\title{
Remisión a largo plazo de la hipertensión portopulmonar mediante trasplante hepático en paciente con cirrosis asociada a hepatitis autoinmune
}

\author{
Long term remission of portopulmonary hypertension with liver transplantation \\ in a patient with cirrhosis associated to autoimmune hepatitis
}

\author{
Alejandro Costaguta ${ }^{(\mathbb{D}}$, María G. Gutiérrez ${ }^{a}$, Guillermo Costaguta ${ }^{a}\left(\mathbb{D}\right.$, Natalia Reyes $^{a}$, Lisandro Bitetti ${ }^{a}$
}

\section{RESUMEN}

La hipertensión portopulmonar (HTPP) es una complicación infrecuente de la hipertensión portal, que sigue un curso progresivo con un pronóstico sombrío. Los reportes en pacientes pediátricos son escasos y con períodos de seguimiento cortos. Se describe una paciente con cirrosis descompensada que desarrolló HTPP resuelta mediante trasplante hepático, que permanece asintomática tras diez años de seguimiento.

Palabras clave: hipertensión pulmonar, hipertensión portal, trasplante de hígado, niño.

http: / / dx.doi.org/10.5546/ aap.2022.e17

Texto completo en inglés:

http: / / dx.doi.org/10.5546/ aap.2022.eng.e17

Cómo citar: Costaguta A, Gutiérrez MG, Costaguta G, Reyes N, Bitetti L. Remisión a largo plazo de la hipertensión portopulmonar mediante trasplante hepático en paciente con cirrosis asociada a hepatitis autoinmune. Arch Argent Pediatr 2022;120(1):e17-e20.

\section{INTRODUCCIÓN}

La hipertensión portopulmonar (HTPP) es una consecuencia de la hipertensión portal descripta tanto en pacientes cirróticos como en no cirróticos. Su detección se ve dificultada porque habitualmente evoluciona de manera silenciosa durante años, aún en estadios avanzados de la enfermedad. ${ }^{1}$ Esta enfermedad sigue un curso progresivo que va desde la vasoconstricción hasta

a. Unidad de Hígado y Trasplante Hepático, Sanatorio de Niños, Rosario, Argentina.

\section{Correspondencia:}

Guillermo Costaguta: gcostaguta5@gmail.com

Financiamiento: Ninguno.

Conflicto de intereses: Ninguno que declarar.

Recibido: 25-7-2020

Aceptado: 7-7-2021 la hipertrofia de las arteriolas pulmonares, lo que lleva a la falla cardíaca derecha. ${ }^{2,3}$ Sin tratamiento, se estima una supervivencia de 6 meses a partir del diagnóstico, y entre $40 \%$ y $70 \%$ a 5 años, aún con tratamiento con vasodilatadores y trasplante hepático (TH). Si bien los casos de HTPP leve a moderada pueden responder favorablemente al TH, el momento óptimo para su indicación no está definido claramente para la población pediátrica, y los reportes son escasos, con poca información sobre el seguimiento a largo plazo. ${ }^{4,5}$

Se describe el caso de una adolescente que se presentó a la consulta con cirrosis descompensada por hepatitis autoinmune descompensada e HTPP moderada, tratada con vasodilatadores y $\mathrm{TH}$, que alcanzó la remisión sostenida a 10 años de seguimiento.

\section{CASO CLÍNICO}

Una paciente de 12 años fue derivada por distensión abdominal dolorosa, ictericia y coluria de cinco días de evolución. Como único antecedente presentaba una apendicectomía a los 8 años, sin mayores complicaciones. A su ingreso impresionaba enferma, con una presión arterial de $110 / 80 \mathrm{mmHg}$, frecuencia cardíaca de 124 lat/min y desdoblamiento del segundo ruido cardíaco. El abdomen era doloroso, con matidez desplazable y una hernia umbilical de reciente aparición. Presentaba, además, edemas periféricos, sin otros hallazgos patológicos.

Los estudios de laboratorio mostraron un compromiso grave de la función hepática (Tabla 1). La radiografía de tórax evidenciaba cardiomegalia a expensas del ventrículo derecho, un arco pulmonar prominente y congestión perihiliar. La ecografía abdominal mostraba un hígado pequeño y heterogéneo, con Doppler normal y ascitis grave; en el ecocardiograma se observaba dilatación atrioventricular derecha con movimiento septal paradojal. La presión sistólica de la arteria pulmonar (PSAP) calculada 
era de $87 \mathrm{mmHg}$ (valor normal [VN]: 18 a $25 \mathrm{mmHg}$ ). Se realizó paracentesis abdominal y se obtuvo líquido con 2200 polimorfonucleares/ $\mathrm{ml}$. Se envió a cultivo, en el que aisló E. coli. Se indicó cefotaxima, furosemida, espironolactona y albúmina por vía intravenosa. Con diagnóstico de hepatitis autoinmune de tipo 1 (IgG: $2896 \mathrm{mg} / \mathrm{dl}$ y anticuerpos antimúsculo liso: 1/640), comenzó tratamiento con prednisona oral (40 mg/día) y sildenafil ( $3 \mathrm{mg} / \mathrm{kg} /$ día). Pasados 15 días de tratamiento, el ecocardiograma mostró una PSAP de $50 \mathrm{mmHg}$ (respuesta parcial), por lo que se decidió realizar un cateterismo cardíaco que mostró una presión media en la arteria pulmonar

TABLA 1. Laboratorio al ingreso y al momento de inscripción en lista de espera de trasplante hepático

\begin{tabular}{lcc}
\hline Parámetros de laboratorio & Admisión & Inscripción \\
\hline Hematocrito $(\%)$ & 37,6 & 32 \\
Hemoglobina $(\mathrm{g} / \mathrm{dl})$ & 12,7 & 10,5 \\
Glóbulos blancos $\left(/ \mathrm{mm}^{3}\right)$ & 9100 & 8700 \\
Plaquetas $\left(/ \mathrm{mm}^{3}\right)$ & 105000 & 92000 \\
Urea $(\mathrm{mg} / \mathrm{dl})$ & 12 & 16 \\
Na $(\mathrm{mEq} / \mathrm{L})$ & 138 & 131 \\
AST $(\mathrm{UI} / \mathrm{L})$ & 328 & 292 \\
ALT $(\mathrm{UI} / \mathrm{L})$ & 279 & 256 \\
Bilirrubina total $(\mathrm{mg} / \mathrm{dl})$ & 3,8 & 3,9 \\
Bilirrubina directa $(\mathrm{mg} / \mathrm{dl})$ & 1,4 & 1,6 \\
Albúmina $(\mathrm{g} / \mathrm{dl})$ & 2,4 & 2,2 \\
RIN & 2,6 & 2,1 \\
\hline
\end{tabular}

AST:aspartatoaminotransferasa,ALT:alanina aminotransferasa, RIN: razón internacional normalizada.
(PmAP) de 36 mmHg (VN: $<25 \mathrm{mmHg}$ ), una resistencia vascular pulmonar (RVP) de 573 dyn. sec.cm ${ }^{5}(7 \mathrm{uW})(\mathrm{VN}:<3 \mathrm{uW})$, y una presión de enclavamiento pulmonar de $12 \mathrm{mmHg}$, congruentes con el diagnóstico de hipertensión pulmonar (HTP) precapilar.

A pesar de la respuesta inicial, 10 días después la paciente presentó una descompensación, con recurrencia de ascitis refractaria al tratamiento y necesidad de cuidados continuos en terapia intensiva; ingresó a lista de espera de TH con un puntaje MELD (modelo para enfermedad hepática terminal, por su sigla en inglés) de 24 , y 24 horas más tarde recibió un órgano completo AB0-idéntico de donante cadavérico. Durante el procedimiento, la PmAP se mantuvo entre 36 y $45 \mathrm{mmHg}$ (medida con un catéter SwanGanz colocado a tal efecto), con buena función del ventrículo derecho. A las dos horas de su ingreso a la unidad de cuidados intensivos pediátricos, la PmAP aumentó a $54 \mathrm{mmHg}$ (más del $50 \%$ de la presión sistémica) sin respuesta al tratamiento conservador, por lo que se comenzó con óxido nítrico inhalado a 20 ppm, que se aumentó a 40 ppm a las siete horas tras un pico de PmAP de $64 \mathrm{mmHg}$. Durante las siguientes horas, los valores de PmAP se estabilizaron alrededor de $40 \mathrm{mmHg}$ y se consiguió el retiro progresivo del óxido nítrico a las 72 horas con una PmAP en torno a los $35 \mathrm{mmHg}$ (Figura 1). La terapia inmunosupresora inicial consistió en metilprednisolona y basiliximab, con el agregado de tracrolimús al cuarto día del

FIGURA 1. Valores de presión media de la arteria pulmonar observados durante el perioperatorio

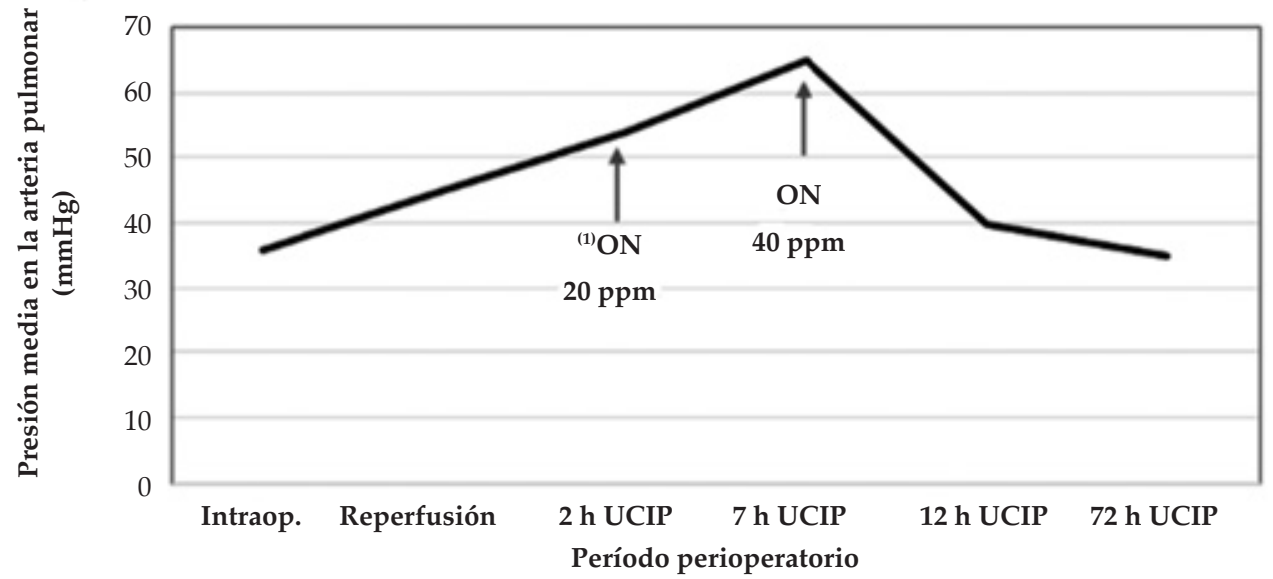

ON: óxido nítrico, ppm: partes por millón, UCIP: unidad de cuidados intensivos pediátricos. 
posoperatorio. Una semana después presentó un episodio convulsivo tratado con antiepilépticos y diagnosticado como encefalopatía posterior reversible secundaria a toxicidad por tacrolimús, por lo que se suspendió su administración en forma transitoria. El día 20 del posoperatorio la paciente fue externada con prednisona (2 $\mathrm{mg} / \mathrm{kg} /$ día), tacrolimús (dosajes de $8 \mathrm{ng} /$ $\mathrm{mL})$ y micofenolato de mofetilo $\left(600 \mathrm{mg} / \mathrm{m}^{2}\right)$. Para el control de la hipertensión pulmonar se mantuvieron dosis de sildenafil de 0,5-1 mg/ $\mathrm{kg} /$ día. Durante los meses siguientes, la PSAP calculada se mantuvo entre 40 y $50 \mathrm{mmHg}$ y la paciente pudo retornar a sus actividades previas sin restricciones. En un intento de normalizar las presiones pulmonares se adicionó bosentán, pero debió interrumpirse debido a un aumento de las enzimas hepáticas (alanina aminotransferasa: 240 UI/L, aspartato aminotransferasa: 198 UI/L), cuyos valores se normalizaron tras la suspensión.

El sildenafil fue suspendido tras 2 años de tratamiento con valores estables de PSAP en los ecocardiogramas.

Durante los años siguientes, la paciente no presentó complicaciones, completó su formación escolar y hoy en día lleva una vida adulta normal. día diez años de recibir el trasplante, se encuentra en tratamiento únicamente con tacrolimús (niveles estables en sangre entre 3-5 ng/ $\mathrm{mL}$ ) y no recibe medicación cardiológica.

\section{DISCUSIÓN}

La HTPP es una condición grave en la que se desarrolla vasculopatía pulmonar progresiva, consecuencia de la desviación portosistémica de sangre esplácnica, tanto en pacientes cirróticos como en no cirróticos. Comienza con una fase de vasoconstricción potencialmente reversible, que progresa hasta una etapa de remodelación arterial con proliferación de la íntima e hipertrofia de la media, probablemente irreversible. Debido a que el tratamiento apropiado puede evitar la progresión, el diagnóstico temprano es mandatorio.

Desafortunadamente, la posibilidad de realizar un diagnóstico oportuno se ve afectada por dos razones principales: primero, la HTPP es infrecuente, con una prevalencia de $5 \%$ entre adultos cirróticos pero menos del $1 \%$ en niños; segundo, dado su naturaleza oligosintomática, la HTPP puede evolucionar durante años sin ser detectada. ${ }^{5}$ Además, la presencia de HTPP no se correlaciona con la gravedad de la enfermedad hepática ni con el grado de hipertensión portal. ${ }^{6}$
Por tanto, su búsqueda sistemática en aquellos pacientes en riesgo es obligatoria.

El diagnóstico de HTPP depende de la demostración de HTP en un paciente con derivación portosistémica de sangre proveniente del lecho esplácnico. Puede sospecharse mediante un ecocardiograma Doppler con una PSAP calculada mayor a $50 \mathrm{mmHg}$ y confirmarse por cateterismo cardíaco con una PmAP mayor a 25 $\mathrm{mmHg}$, una resistencia superior a 3 unidades Wood (240 dyn.sec.cm ${ }^{-5}$ ), y una presión de enclavamiento pulmonar menor a $15 \mathrm{mmHg}{ }^{1,4,5}$ El enfoque inicial es el tratamiento con vasodilatadores y antagonistas de los receptores de la endotelina, pero la resolución definitiva solo puede alcanzarse mediante la eliminación de la causa, que en pacientes cirróticos implica un TH. Sin embargo, la selección tanto del candidato como del momento debe realizare en forma cuidadosa. ${ }^{7,8}$

La HTPP fue considerada durante mucho tiempo una contraindicación clara para el TH; sin embargo, un mejor conocimiento de la enfermedad y los buenos resultados reportados en casos aislados han hecho que hoy en día sea una indicación posible para el TH. Con base en los valores de PmAP obtenidos mediante cateterización cardíaca derecha, puede clasificarse en leve (25-34 mmHg), moderada (35-44 $\mathrm{mmHg}$ ) o grave $(\geq 45 \mathrm{mmHg}){ }^{5}$ Los pacientes con buena respuesta a los vasodilatadores son considerados buenos candidatos para $\mathrm{TH}$ y deben inscribirse en una lista con consideraciones especiales en sus puntajes de MELD/PELD (enfermedad hepática terminal pediátrica, por su sigla en inglés). 4,7,9 Constatar una función ventricular derecha adecuada mediante ecocardiograma Doppler transtorácico puede contribuir a la toma de decisión en casos difíciles. ${ }^{10}$

Si bien la reversión de la HTPP con vasodilatadores y $\mathrm{TH}$ ha sido reportada previamente, ${ }^{11,12}$ la serie más grande de pacientes pediátricos consiste en 14 casos de los cuales solo 9 pacientes presentaban cirrosis, y todos secundarios a atresia de vías biliares, un escenario muy diferente a la cirrosis posnecrótica del caso aquí presentado. ${ }^{4}$ Dos de los pacientes fallecieron de manera precoz, por lo que únicamente pudieron reportarse 7 niños con resolución de la HTPP, y solo 3 de ellos tuvieron un seguimiento mayor a dos años.

La paciente presentada experimentó una mejoría rápida y un desenlace favorable posterior al $\mathrm{TH}$, regresando a su vida normal unos meses 
después de la cirugía. Dos años más tarde, se suspendió la administración de sildenafil, y los valores de PSAP se mantuvieron dentro de parámetros normales.

En resumen, este caso clínico ilustra una evolución favorable de HTPP secundaria a cirrosis posnecrótica, con remisión en el largo plazo tras diez años de seguimiento. Esto destacan el valor del TH oportuno como indicación en pacientes cuidadosamente seleccionados.

\section{REFERENCIAS}

1. Rodríguez-Roisin R, Krowka M, Hervé P, Fallon M, et al. Pulmonary-Hepatic vascular disorders (PHD). Eur Respir J. 2004; 24(5):861-80.

2. Cartin-Ceba R, Krowka M. Portopulmonary hypertension. In Lee S, Moreau R, (eds). Cirrhosis. A practical guide to management. West Sussex: John Wiley \& Sons; 2015. Págs.212-24.

3. Swanson K, Krowka M. Pulmonary complications associated with portal hypertension. In: Sanyal A, Shah V, (eds). Portal Hypertension. Pathobiology, evaluation, and treatment. New Jersey: Humana Press; 2010.Págs.455-68.

4. Ecochard-Dugelay E, Lambert V, Schleich J, Duché M, et al. Portopulmonary hypertension in liver disease presenting in childhood. J Pediatr Gastroenterol Nutr. 2015; 61(3):34654 .
5. LeeW, WongS, Ivy D, SokolR. Hepatopulmonary syndrome and portopulmonary hypertension in children: recent advances in diagnosis and management. J Pediatr. 2018; 196:14-21.e1.

6. Raevens S, Colle I. Hepatopulmonary Syndrome and Portopulmonary Hypertension. In Muriel P (ed). Liver Pathophysiology: Therapies and Antioxidants. London: Elsevier; 2017.Págs.517-22.

7. Sokol R. Portopulmonary hypertension: opportunities for precision pediatrics. J Pediatr Gastroenterol Nutr. 2015; 61(3):268-9.

8. ErikssonC,GustavssonA,KronvallT,TyskC.Hepatotoxicity bybosentan in a patient with portopulmonary hypertension: a case-report and review of the literature. J Gastrointestin Liver Dis. 2011; 20(1):77-80.

9. Krowka M, Fallon M, Mulligan D, Gish R. Model for endstage liver disease (MELD) exception for portopulmonary hypertension. Liver Transpl. 2006; 12(Suppl 3):S114-6.

10. Ramsay M. Portopulmonary hypertension and right heart failure in patients with cirrhosis. Curr Opin Anaesthesiol. 2010; 23(2):145-50.

11. Laving A, Khanna A, Rubin L, Ing F, et al. Successful liver transplantation in a child with severe portopulmonary hypertension treated with epoprostenol. J Pediatr Gastroenterol Nutr. 2005; 41(4):466-8.

12. Salgia R, Goodrich N, Simpson H, Merlon R, Sharma P. Outcomes of liver transplantation for porto-pulmonary hypertension in model for end-stage liver disease era. Dig Dis Sc. 2014; 59(8):1976-82. 